\title{
Regions of High Dominant Frequency in Chronic Atrial Fibrillation Anchored to Areas of Atrial Fibrosis
}

\author{
Nathan Angel ${ }^{1,2}$, Eugene G Kholmovski ${ }^{3,4}$, Elyar Ghafoori ${ }^{1,2,5}$, Derek J Dosdall ${ }^{2,5,6}$, \\ Rob S MacLeod ${ }^{1,2,5}$, Ravi Ranjan ${ }^{1,2,5}$
}

\begin{abstract}
From the ${ }^{1}$ Division of Cardiovascular Medicine, ${ }^{2}$ Department of Biomedical Engineering, ${ }^{3}$ UCAIR, Department of Radiology and Imaging Sciences, ${ }^{4}$ CARMA Center, ${ }^{5}$ Nora Eccles Harrison Cardiovascular Research and Training Institute, and ${ }^{6}$ Department of Surgery, University of Utah, Salt Lake City, UT, United States of America
\end{abstract}

\begin{abstract}
Regions within the atria with sustained rapid reentrant or focal activity have been defined as a mechanism of persistent atrial fibrillation (AF). However, the mechanism behind the anchoring of these sites and their stability over time is unknown. We tested the hypothesis that fibrosis anchors sites of high frequency activation during $A F$ and that these sites can be non-invasively determined using cardiac T1 Mapping with MRI.

$A$ canine rapid atrial paced model of persistent $A F$ was used ( $n=12$, including 6 controls) for the study. Whole heart TI Mapping was performed prior to an electrical mapping study. Spatial maps of high dominant frequency (DF) probability were constructed to determine stability of the highest DF sites. These sites were then correlated with fibrotic regions determined by T1 Mapping.

The chronic AF animals had at least one site of stable, high DF for at least 22.5 (75\%) of 30 minutes of $A F$. Regions of stable high DF bordered regions of fibrosis as determined by T1 Mapping MRI $82 \%$ of the time $(p<0.05)$.

Heterogeneous atrial remodeling, specifically fibrosis, arising from chronic AF may provide a substrate that anchors sites of high DF. Cardiac T1 Mapping with MRI may determine such sites non-invasively.
\end{abstract}

\section{Introduction}

Localized regions within the atria have been implicated in supporting rapid and organized activations during $\mathrm{AF}$, which, in turn may promote this arrhythmia[1]. However, the stability of these sites over prolonged recording periods remains controversial and it is not clear if there is a structural component to the cardiac substrate that may, at least transiently, stabilize rapid foci or reentrant activity to a location.

Myocardial fibrosis is a well-established marker of adverse structural remodeling in a variety of heart diseases including $\mathrm{AF}[2]$ and magnetic resonance imaging (MRI) provides a means to visualize fibrosis. Specifically, post contrast T1 Mapping with MRI, is one of the few validated non-invasive methods for determining cardiac fibrosis[3]. We hypothesis that regions of the atria with significant fibrotic structural remodeling can anchor, regions of rapid focal or reentrant activity, which drive $\mathrm{AF}$, and that cardiac post contrast $\mathrm{T} 1$ Mapping with MRI can non-invasively locate these sites.

\section{Methods}

All animals were managed in accordance with the Guide for the Care and Use of Laboratory Animals[4], and the protocol was approved by the Institutional Animal Care and Use Committee of the University of Utah.

\subsection{Experimental preparation}

Animal Model: Mongrel dogs ( $\mathrm{n}=12)$, weighting 25-35 $\mathrm{kg}$ were used for this study. Neurostimulators (Itrels, Medtronic Inc.) were implanted in 6 of the dogs and chronic AF was induced with rapid atrial pacing (RAP). The other 6 dogs were used as a baseline for the electrophysiological study and were not evaluated with MRI. Procedures for the pacemaker implantation and programming have been described in detail previously[5].

\subsection{MRI Study}

In all of the chronic AF dogs, the cardiac substrate was evaluated with high-resolution, whole heart T1 Mapping with MRI at baseline, before the RAP began. The chronic AF dogs were again evaluated with whole heart $\mathrm{T} 1$ Mapping after $18 \pm 3$ months of AF and 1-3 weeks prior to a detailed electrophysiological study.

\subsection{MRI Acquisition and Processing}

Whole heart T1 Mapping with MRI was performed on a 3 Tesla whole-body Verio and Trio MRI scanners 
(Siemens Healthcare) during slow infusion of contrast agent $(0.05 \mathrm{micromol} / \mathrm{kg} / \mathrm{sec}$ of Gd-BOPTA, Bracco Diagnostic Inc.). Initial contrast bolus $(0.15 \mathrm{mmol} / \mathrm{kg})$ was given about 20 minutes prior to the start of the slow infusion. T1 Mapping MRI was performed at least 30 minutes after start of the slow infusion to achieve steadystate of contrast distribution. T1 Mapping was estimated according to the Bloch equation governing $\mathrm{T} 1$ magnetization evolution[6].

The T1 MRI maps were segmented and processed using the Seg3D software package (University of Utah, Salt Lake City, UT) to determine regions of the LA wall with low T1 values. Within the LA wall, regions with the lowest $10 \% \mathrm{~T} 1$ values were considered to contain the largest density of fibrotic tissue. A geometrical shell of the LA wall with regions of fibrosis was exported into an electroanatomical mapping system (EAMS) (EnSite Velocity Electro-anatomical Mapping System, St Jude Medical, Inc, St. Paul, MN, USA), and used to match the fibrotic sites with the high DF sites as described in the next section. For each animal, the average T1 values of the LA wall was computed as well as the average of the fibrotic region as defined from T1 Mapping (lowest 10\% of the T1 times).

\subsection{Electrophysiological Study}

A detailed electrophysiological (EP) study was done in both the controls and in the chronic AF animals. For the chronic AF animals, the EP study was done 1-3 weeks after whole heart T1 mapping and after they had persistent AF for more than 12 months.

\subsection{Data Acquisition and Processing}

For the chronic AF animals, an electrical anatomical map (EAM) of the left atrium (LA) was made and was merged with the Seg3D segmentations of T1 Mapping MRI using the fusion application of the EnSite (St Jude Medical) mapping system. The segmentation contained the LA wall and the regions of fibrosis, as determined by T1 Mapping. A 64-pole basket catheter $(38 \mathrm{~mm}$ for controls and $48 \mathrm{~mm}$ for chronic AF animals) (Constellation, Boston Scientific Corp, Natick, MA, USA) was then placed into the LA. An ECG and endocardial unipolar electrograms from the basket catheter were recorded for up to 30-minutes of AF. AF was induced in the controls with 30 seconds of $50 \mathrm{~Hz}$ burst pacing at $5 \mathrm{~mA}$ from a catheter placed in the right atrium. If AF was not induced or persisted for less than 30 minutes in the controls, burst pacing was reapplied with a maximum of five attempts.

The ECG and unipolar electrograms were sampled at $4096 \mathrm{~Hz}$ with 24-bit resolution with an Active Two mapping system (Active Two System, Biosemi, Inc,
Amsterdam, Netherlands). The acquired signals were then preprocessed with custom MATLAB (Mathworks, Inc, Natick, MA, USA). Far-field ventricular activity was reduced using a template based QRS subtraction algorithm. The resulting unipolar atrial electrograms were then analyzed with DF spectral analysis. The dominant frequency for each basket electrode was computed for every minute of AF. High DF sites were determined as those with DFs in the highest $10 \%$ for each minute of AF, and had to have DFs at least $1 \mathrm{~Hz}$ higher than the average of the DF from the rest of the LA. The high DF sites for each minute of AF were placed in a spatial map of probability to determine stability of the sites during the 30 min AF episode. Stable sites of high DF were determined as an electrode site with $75 \%$ or higher stability over the time course of the AF episode. Each electrode location was projected onto the closest location on the LA wall segmentation, such that the DF values could be directly compared to the $\mathrm{T} 1$ times. Electrode positions greater than $10 \mathrm{~mm}$ from the LA wall were excluded from all analysis. The LA wall was assigned a binary value of whether it was a region of healthy or fibrotic tissue, where fibrotic regions were defined to be within $2 \mathrm{~mm}$ of the lowest $10 \%$ T1 values over the entire LA wall. Every stable high DF site for each animal was then classified as either in a fibrotic or healthy region. For each chronic AF animal, the percentage of the number of stable high DF sites that were on the border zones was then computed.

\subsection{Histology}

Histological tissue samples, each with surface area of approximately 1-3 $\mathrm{mm}^{2}$, taken from the chronic AF hearts were stained with Masson's trichrome. Multiple such samples were collected from regions of fibrosis determined from T1 Mapping and from healthy areas of the LA of the same animals. Images from the samples were imported into ImageJ software (National Institutes of Health) for fibrosis quantification.

\subsection{Statistical analysis}

The paired $t$-test was used to analyze continuous variables and a chi-square test was used to determine whether regions of stable high DF were observed in the fibrotic regions more often than in healthy regions. The expected value was the percentage of the electrodes that were within $2 \mathrm{~mm}$ of fibrosis. Results were considered significant at $\mathrm{p}<0.05$.

\section{Results}

T1 relaxation times in the atrial wall significantly shortened following chronic AF (after $18 \pm 3$ months of AF) (Baseline before AF: $593 \pm 41 \mathrm{~ms}$ vs. Chronic AF: 
$463 \pm 79 \mathrm{~ms}, \mathrm{p}<0.01, \mathrm{n}=5$, lower $\mathrm{T} 1$ time corresponds to larger extracellular space). In the chronic AF animals, the regions of lowest $10 \% \mathrm{~T} 1$ values had an average $\mathrm{T} 1$ time of $283 \pm 32 \mathrm{~ms}(\mathrm{n}=6)$ and these regions were in patches and not diffusely distributed throughout the LA wall. Regression analysis showed an inverse relationship between T1 time and the histological fibrosis percentage (Figure 1) with a $\mathrm{R}^{2}$ of 0.23 and the $\mathrm{p}<0.01(\mathrm{n}=68)$. These histological samples grouped across all six of the chronic $\mathrm{AF}$ animals were quantified for \% fibrosis and confirmed that regions of T1 Mapping detected fibrosis had higher fibrosis percentages as compared to other LA regions in the chronic $\mathrm{AF}$ animals $(19.0 \pm 7.5 \%$ vs. $10.5 \pm 4.0 \%, \mathrm{p}<0.05, \mathrm{n}=6$ ).

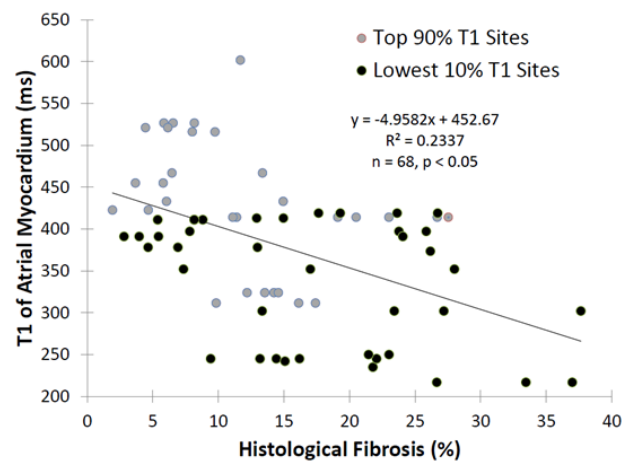

Figure 1. Post contrast T1 Mapping MRI detected Fibrosis agrees with Histology. Histological determined fibrosis percentage plotted against the T1 time.

All of the chronic AF animals could sustain AF for the full 30 minutes, whereas none of the controls had AF over 14 minutes. The controls did not have any stable, high DF sites while each chronic AF animal had at least one site of stable, high DF for at least 22.5 (75\%) of 30 minutes of $\mathrm{AF}$. The mean DF in the chronic AF animals was $8.5 \pm 1.2$ $\mathrm{Hz}$ and the stable high DF sites had a mean DF of $10.3 \pm 1.1 \mathrm{~Hz}(\mathrm{p}<0.01, \mathrm{n}=6)$. The sites of stable, high DF for a single AF episode may vary in DF by as much as 1 $\mathrm{Hz}$ throughout the 30 minute AF episode; however, these sites still maintained higher DF's than other nearby locations, suggesting these sites have more spatial stability than temporal stability (Figure 2).
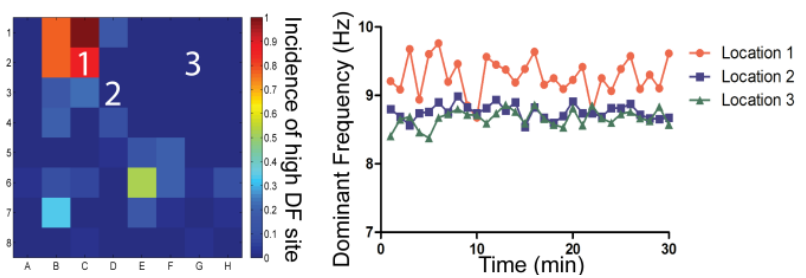

Figure 2. Stability of high DF sites in chronic AF. An example of spatial stability of high DF sites over 30minute time course of AF. $\mathrm{X}$ axis shows the spline numbers $\mathrm{A}-\mathrm{H}$ and $\mathrm{Y}$ axis shows electrode numbers on the corresponding spline. Each electrode sites contains the incidence that it had a DF value within the top $10 \%$ over the AF episode. DF values for 3 locations, labeled 1-3, are shown over the time course of a 30 min AF episode.

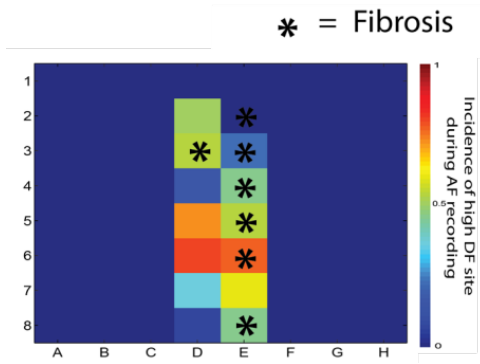

Figure 3. Relationship between regions of high DF and fibrosis. Example of a spatial probability plots of DF and regions of fibrosis. $\mathrm{X}$ axis shows the spline numbers A-H and $\mathrm{Y}$ axis shows electrode numbers on the corresponding spline. Each electrode sites contains the incidence that it had a DF value within the top $10 \%$ over the atrial fibrillation episode.

Table 1. Summary of Electrophysiological Study

\begin{tabular}{cccccc}
\hline $\begin{array}{c}\text { AF } \\
\text { animal } \\
\text { number }\end{array}$ & $\begin{array}{c}\text { Number } \\
\text { of high DF } \\
\text { sites }\end{array}$ & $\begin{array}{c}\text { Incidence of } \\
\text { high DF sites } \\
\text { bordering fibrosis } \\
(\%)\end{array}$ & $\begin{array}{c}\text { Average } \\
\text { of stable } \\
\text { high DF } \\
\text { values (Hz) }\end{array}$ & $\begin{array}{r}\text { Average } \\
\text { DF value in } \\
\text { all of the LA } \\
(\mathrm{Hz})\end{array}$ & $\begin{array}{c}\text { Fibrotic } \\
\text { border zone } \\
(\%)\end{array}$ \\
\hline 1 & 4 & $75 \%$ & 9.3 & 8.2 & $33 \%$ \\
2 & 3 & $67 \%$ & 11.4 & 9.5 & $47 \%$ \\
3 & 1 & $100 \%$ & 10.9 & 9.7 & $52 \%$ \\
4 & 4 & $50 \%$ & 10.7 & 7.0 & $66 \%$ \\
5 & 1 & $100 \%$ & 11.0 & 9.2 & $22 \%$ \\
6 & 2 & $100 \%$ & 8.7 & 7.2 & $56 \%$ \\
Average & 2.5 & $82 \%$ & 10.3 & 8.5 & $46 \%$ \\
\hline
\end{tabular}


In the chronic $\mathrm{AF}$ animals, $\sim 50 \%$ of the mapped region occurred on the border regions of fibrosis however, on average $82 \%$ of stable high DF bordered regions of fibrosis as determined by T1 Mapping, indicating that high DF sites preferentially form in the border regions of fibrosis (Figure 3). High DF sites were observed in 15 locations with 384 total locations recorded with the constellation catheter. Of the 15 high DF sites, 11 bordered a region of fibrosis. A chi square test confirmed that the high DF sites preferentially occurred in the border regions of fibrosis as compared to non-fibrotic regions ( $p$ $<0.05$ ). The average separation between the stable high DF sites and the closest region of fibrosis was $1.4 \pm 1.2$ $\mathrm{mm}$. Table 1 summarizes the results of the EP/MRI study. The fibrotic regions consisted of a large area of the basket mapping field in some animals; this was primarily due to the constraint that if a basket could be placed in multiple locations due to the large LA size, the location with the highest DF was used for the analysis.

\section{Discussion}

The major findings of this study were as follows: 1) High resolution T1 Mapping identified LA wall areas with fibrotic tissue. 2) During chronic AF, but not in controls, there were regions within the LA that had stable sites of high DF over a prolonged recording period. 3) The sites of stable high DF anchored to fibrotic regions.

T1 Mapping can measure diffuse fibrosis by quantifying absolute values of the T1 relaxation time for each voxel. We used this feature of T1 mapping in our study to show that in animals with chronic AF induced with RAP, T1 times significantly decreased indicating that atrial structural remodeling was occurring. Furthermore, the chronic AF animals had specific regions within the LA with more fibrotic remodeling than other regions.

Our findings indicate that during chronic $\mathrm{AF}$, but not in controls, there are sites within the LA that anchor rapid activation. The controls either did not sustain AF or, in the one case that it did, the high DF sites were not stable, which suggest that the stability of localized driving sites may require the development of fibrotic atrial remodeling. The sites of localized stable high DF tend to anchor to the most fibrotic regions in the left atrium.

There is much growing evidence that driving sites during some forms of persistent $\mathrm{AF}$ are associated with fibrotic tissue. Jadidi et al. found that that sites of complex fractionated atrial electrograms (CFAEs) tended to occur on sites of dense fibrotic tissue as determined by delayed enhancement (LGE-) MR imaging[7]. Hansen et al. found that intramural reentry, as determined by optical mapping, was facilitated by fibrotic-insulated atrial bundles, as determined by ex-vivo MR imaging[8]. A major barrier to understanding the role of fibrosis and $\mathrm{AF}$ mechanisms is that non-invasive methods of fibrosis detection are difficult to validate. Many studies use indirect measurements of fibrosis, some of which are electrophysiological, such as regions of low amplitude or complex fractionation, others use MR imaging, most commonly late gadolinium enhancement (LGE-MRI). We present for the first time results from quantitative T1 mapping of the whole atrium to show progression of structural remodeling and validate this non-invasive measure of fibrosis with histology. The cumulative evidence of these studies suggests that atrial structural remodeling, specifically fibrosis, form arrhythmogenic sites. Moreover, we show that T1 mapping might reliably detect such fibrosis non-invasively.

\section{References}

[1] B. J. Hansen et al., "Atrial fibrillation driven by microanatomic intramural re-entry revealed by simultaneous sub-epicardial and sub-endocardial optical mapping in explanted human hearts," Eur. Heart J., pp. 2390 2401, 2015.

[2] J. Pellman, R. C. Lyon, and F. Sheikh, "Extracellular matrix remodeling in atrial fibrosis: mechanisms and implications in atrial fibrillation.," J. Mol. Cell. Cardiol., vol. 48, no. 3, pp. 461-7, Mar. 2010.

[3] C. McGann et al., "Atrial fibrillation ablation outcome is predicted by left atrial remodeling on MRI," Circ. Arrhythmia Electrophysiol., vol. 7, no. 1, pp. 23-30, 2014.

[4] National Research Council, Guide for the Care and Use of Laboratory Animals, 8th ed., vol. 66. 1996.

[5] D. J. Dosdall et al., "Chronic atrial fibrillation causes left ventricular dysfunction in dogs but not goats: experience with dogs, goats, and pigs.," Am. J. Physiol. Heart Circ. Physiol., vol. 305, no. 5, pp. H725-31, Sep. 2013.

[6] M. Fitts et al., "Arrhythmia insensitive rapid cardiac T1 mapping pulse sequence.," Magn. Reson. Med., vol. 70, no. 5, pp. 1274-82, Dec. 2013.

[7] A. S. Jadidi et al., "Inverse relationship between fractionated electrograms and atrial fibrosis in persistent atrial fibrillation: combined magnetic resonance imaging and high-density mapping.," $\mathrm{J}$. Am. Coll. Cardiol., vol. 62, no. 9, pp. 802-12, Aug. 2013.

[8] B. J. Hansen et al., "Atrial fibrillation driven by microanatomic intramural re-entry revealed by simultaneous sub-epicardial and sub-endocardial optical mapping in explanted human hearts," Eur. Heart J., vol. 36, no. 35, pp. 2390-2401, 2015.

Address for correspondence:

Ravi Ranjan, MD PhD

Division of Cardiovascular Medicine

University of Utah

30 North 1900 East, Rm 4A100

Salt Lake City, UT 84132

ravi.ranjan@hsc.utah.edu 